\title{
VMAT vs Eight Field Imrt: Dosimetric Comparison of Pelvic Radiotherapy for Patients with High-Risk Prostate Cancer in Terms of Bone Marrow Sparing
}

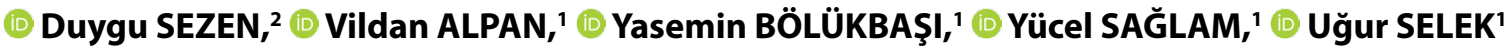

'Department of Radiation Oncology, Koç University School of Medicine, İstanbul-Turkey

${ }^{2}$ Department of Radiation Oncology, American Hospital-Koç University Hospital, İstanbul-Turkey

\section{OBJECTIVE}

Although there is no complete consensus on elective pelvic nodal irradiation for patients with high-risk prostate cancer, pelvic radiotherapy with androgen ablation has been more commonly used in many centers. An important part of bone marrow (BM) reserve remains in the pelvic radiation treatment field. This study aimed to evaluate and compare the intensity modulated (step-and-shoot IMRT: ssIMRT) and volumetric modulated arc radiotherapy (VMAT) techniques for pelvic radiotherapy in terms of pelvic BM doses.

\section{METHODS}

This study was based on the simulation scan data of 10 patients with prostate cancer as 3-mm slice thickness using a full bladder and rectal balloon. The first phase of the treatment planning prescribed to pelvic lymphatic (46 Gy/2 Gy/fraction). The second phase consisted of the seminal vesicles and prostate ( $32 \mathrm{~Gy} / 2 \mathrm{~Gy} /$ fraction). The PTV margin was $0.4 \mathrm{~cm}$ posteriorly due to rectum and $0.6 \mathrm{~cm}$ in all other (including $\left.\mathrm{PTV}_{\text {lymphatic }}\right)$ directions. Using same target volumes, ssIMRT with eight angles $\left(225^{\circ}, 260^{\circ}\right.$, $\left.295^{\circ}, 330^{\circ}, 30^{\circ}, 65^{\circ}, 100^{\circ}, 135^{\circ}\right)$ and double arc $\left(182^{\circ},-178^{\circ}\right.$ arc angle) VMAT were planned for each patient data set. The planning objective was to cover the PTV by at least $95 \%$ of the prescribed isodose and CTV by $98 \%$ of the prescribed isodose line. No special dose constraint was given for BM sparing. Each technique was compared by using dose volume histograms (DVH) of V5, V10, V20, V30, V40 of the sacral BM (SBM), iliac BM (IBM), and ischium, pubis, and proximal femora (lower pelvis) and femoral BM (FBM). In addition, V20 V30, V40, and V70 for bladder, and V30, V40, V76, and V80 for the rectum, homogeneity index and the monitor units (MU) were evaluated. The two-sided Wilcoxon's test was used for statistical analysis $(\mathrm{p}<0.05)$.

\section{RESULTS}

For the same PTV coverage, VMAT and ssIMRT plans had similar dose distributions for FBM, IBM, SBM, and total BM as well as the other critical structures. However, VMAT plans in comparison to IMRT ensured significantly lower high dose volumes on rectum such as bringing V80 from $1.6 \%$ to $0.9 \%(\mathrm{p}=0.01)$, and provided similar homogeneity index with lowered monitor units (1048 vs. 1591, $\mathrm{p}=0.018$ ).

\section{CONCLUSION}

In this cohort, VMAT plans without a specific constraint for BM are not found to be superior to ssIMRT in terms of BM reserve irradiation; while VMAT could be encouraged for patients with higher rectum doses such as V80.

Keywords: BM; intensity modulated radiotherapy; prostate; pelvic; radiotherapy; volumetric arc radiotherapy. Copyright @ 2019, Turkish Society for Radiation Oncology

Received: June 17, 2017

Accepted: June 24, 2017

Online: August 26, 2019

Accessible online at:

www.onkder.org
Dr. Uğur SELEK

Koç Üniversitesi Tıp Fakültesi,

Radyasyon Onkolojisi,

İstanbul-Turkey

E-mail: ugurselek@yahoo.com 


\section{Introduction}

Prostate only versus whole pelvis RT has long been a debate in management of prostate cancer,[1-4] especially for patients with clinically high-risk or locally advanced who have been diagnosed with localized extensive disease with the presence of invasion of adjacent structures/ extracapsular extension/ seminal vesicle involvement or a serum PSA $>20 \mathrm{ng} / \mathrm{mL}$ or a Gleason score of 8-10 without lymph node involvement or distant metastases. Until the results of phase III RTOG 0924 trial (NCT01368588) will highlight the pathway for a standard of care, most centers continue to offer pelvic radiotherapy for these patients. As a clinic that does not recommend pelvic radiotherapy regardless of the extent of their primary tumor when there is no documented lymph node involvement, we would like to evaluate whether the cutting edge technology effect in BM sparing in case of volumetric arc radiotherapy (VMAT) or intensity modulated radiotherapy (IMRT) used for standard pelvic fields.

Radiotherapy causes myelosuppresion because of apoptosis of bone marrow (BM) stem cells and stromal damage. [5] Considering that approximately $40 \%$ of the total body $\mathrm{BM}$ is located in the pelvic bones involving femur, iliac, and sacrum, BM sparing becomes a raising question to increase treatment tolerability, especially for patients with pelvic malignancies receiving pelvic radiotherapy with concurrent chemotherapy. Therefore, BM sparing was first evaluated in patients with cervical cancer receiving concurrent radiochemotherapy as a standard treatment.[6,7] Compared to conventional techniques, IMRT was found to be superior in terms of BM sparing. As grade 2 or more hematological toxicities were noted to be related with V20 dose of whole pelvic bone that requires further clinical evaluation, [8] a newer study by Liang et al pointed out a functional BM sparing pelvic IMRT technique based on 18F-FDG-PET and quantitative MRI for gynecological and anal canal cancers. [9] Sini et al.[10] prospectively evaluated 121 patients with prostate cancer who underwent adjuvant or salvage WPRT and reported that higher BM V40 was associated with higher acute grade 3 or late grade 2 lymphopenia. In all dosimetric and clinical studies, IMRT was the only treatment planning technique that was used to compare conventional techniques and applied in the clinical routine approach.

Despite ongoing discussions upon prostate only versus whole pelvis RT approach in patients with prostate cancer, these patients with pelvic lymph node metastasis risk greater than $15 \%$ were found to be rationale to be recommended whole pelvic radiotherapy with hormonal treatment.[1] IMRT for pelvic nodal radiotherapy was also noted to promise safer dose delivery and fewer side effects.[11] VMAT has also been shown to provide equal or superior dosimetric dose delivery for critical structures such as rectum, femoral heads, and bladder with an advantage of less treatment time.[12,13] Davidson et al reported a VMAT delivery efficacy with an average $75 \%$ reduction of treatment time and $15 \%-38 \%$ fewer monitor units.[14]

A cone-down technique for a patient with highrisk prostate cancer with a first phase of 46 Gy whole pelvic and then second phase of 30-32 Gy prostate and seminal vesicles irradiation could represent one of the highest prescribed external doses in the pelvic malignancies. In our study, we intended to evaluate and compare the ssIMRT and VMAT techniques for pelvic radiotherapy in terms of pelvic BM doses based on the plans created for the same patients with highrisk prostate cancer and to seek any difference in BM sparing between these two techniques.

\section{Materials and Methods}

The planned computerized tomography (CT) scans of 10 consecutive patients with prostate cancer treated by definitive intent to $78 \mathrm{~Gy}$ in 39 fractions to prostate and seminal vesicles at our institution were retrieved for this institutional review board approved study. The details of simulation, volume definition, planning, and comparison are given below.

\section{Simulation}

All patients were simulated in the supine position in a customized vac loc bed in addition to knee-foot stopper immobilization (CIVCO, Kalona, Iowa). The CT images with 3-mm slice thickness from above the L4 vertebra down to the trochanter minor were obtained with full bladder (200-250 cc) and rectal balloon (Radiadyne, LLC) by the Philips Brilliance Big Bore 16 slice CT (Phillips, Inc.). Bladder scan (Verathon Bladder Scan BVI 6400) was routinely used to ensure full bladder before each treatment.

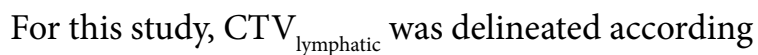
to the Radiation Therapy Oncology Group (RTOG) consensus guidelines.[15] First phase radiotherapy of 46 Gy was prescribed to whole pelvis; then second phase of $32 \mathrm{~Gy}$ was prescribed to prostate and the proximal seminal vesicles that was described as $\mathrm{CTV}_{2}$. According to this study protocol, $\mathrm{CTV}_{1}$ consists of $\mathrm{CTV}_{\text {lymphatic }}$ and $\mathrm{CTV}_{2}$. PTV margin was given as $4 \mathrm{~mm}$ posterior and $6 \mathrm{~mm}$ in other (including $\mathrm{PTV}_{\text {lymphatic }}$ ) directions. 
Normal tissues included bowel, bladder, rectum, and $\mathrm{BM}$ reserve that comprised the sacral BM (SBM), iliac $\mathrm{BM}$ (IBM), and ischium, pubis, and proximal femora (lower pelvis) as femoral BM (FBM). The external contour of the pelvic bones was delineated on CT scan to define the BM, as described previously by Mell et al in the BM sparing of cervix cancer study (Fig. 1).[7] The prescribed total dose was 78 Gy delivered in two phases of 23 and 16 fractions, respectively.

To generate VMAT and IMRT plans for each patient, we used the Pinnacle3 v9.0 treatment planning system (Philips Medical Systems Inc. Cleveland, $\mathrm{OH}$ ) that uses Collapse Cone [cc] Convolution algorithm. The planning objective was to cover the PTV by at least $95 \%$ of the prescribed isodose and CTV by $98 \%$ of the prescribed isodose line. Identical objectives were set for IMRT and VMAT plans. The treatment plans were generated utilizing the $6 \mathrm{MV}$ Photon beam from the Varian Triology 120 leaf millennium multileaf collimator (MLC) (Varian Medical Systems, Palo Alto, CA). We delivered the plans with a maximum dose rate of $600 \mathrm{MU} / \mathrm{min}$. All calculations utilized a calculation grid size of $0.3 \times 0.3 \times 0.3 \mathrm{~cm}$. Figure 2 displays the field arrangements for IMRT and VMAT plans. The rectum
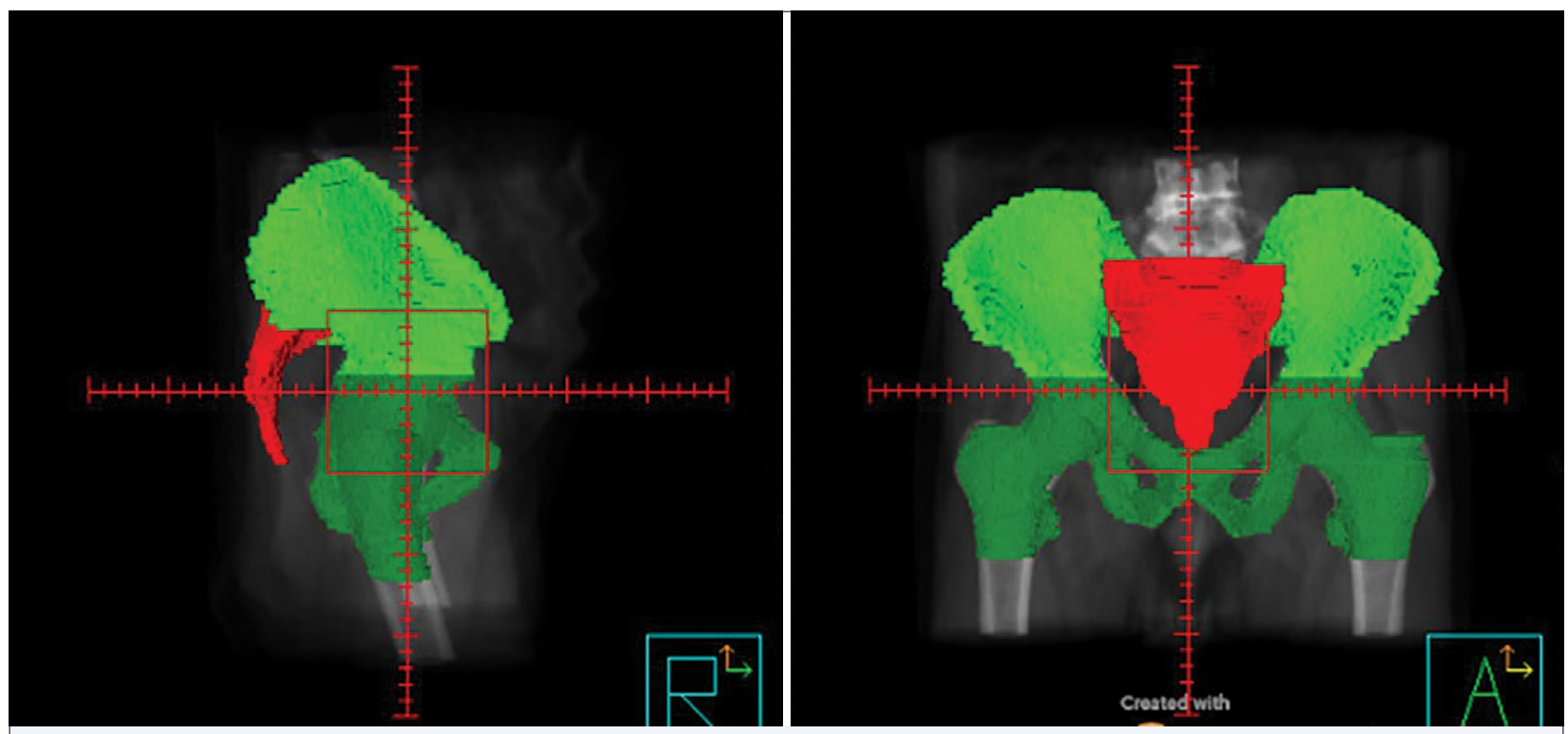

Fig. 1. Anteroposterior and lateral DRRs showing the delineation of sacrum BM (red), iliac BM (light green), and ischium, pubis, and proximal femora (lower pelvis) as femoral BM (dark green).
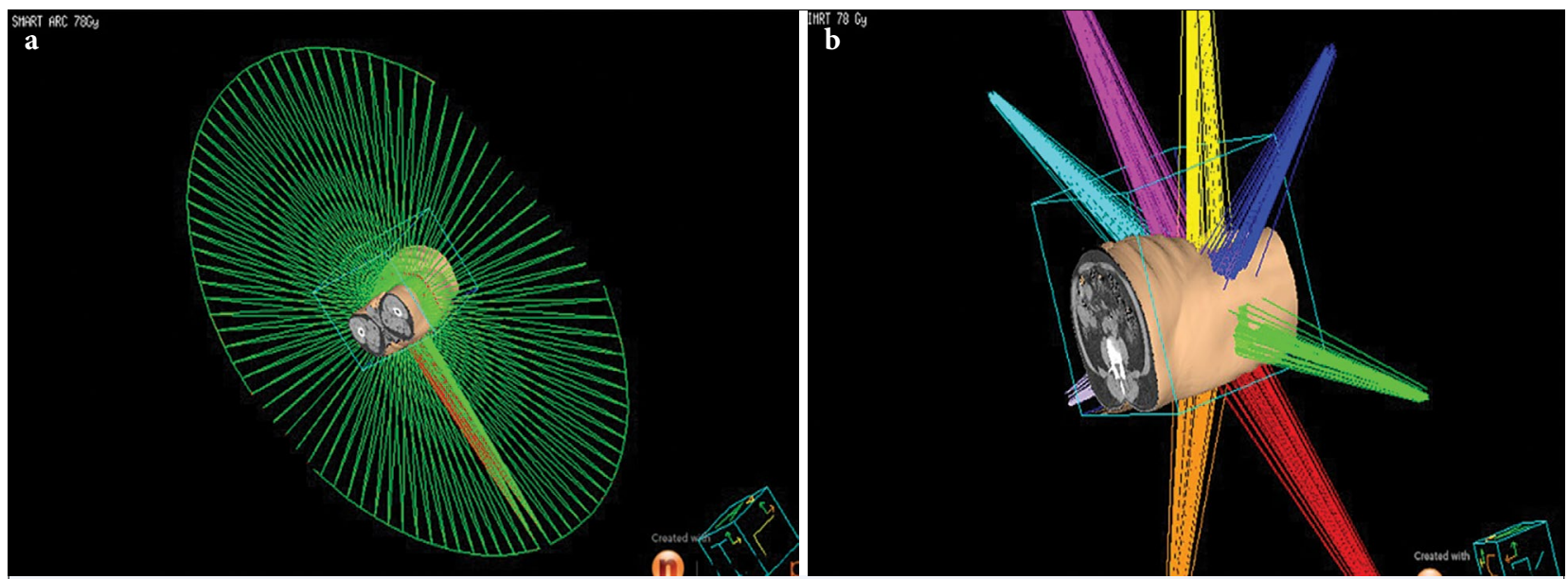

Fig. 2. Field design for (a) VMAT using two $360^{\circ}$ arcs with the same isocenter rotating clockwise and counter-clockwise starting from $182^{\circ}$ and $178^{\circ}$ with a $10^{\circ}$ collimator angle and (b) ssIMRT using eight non-coplanar or coplanar beams $\left(225^{\circ}, 260^{\circ}, 295^{\circ}, 330^{\circ}, 30^{\circ}, 65^{\circ}, 100^{\circ}, 135^{\circ}\right)$. 
planning goals criteria were V $80<5 \%$, V $75-76<15 \%$, 70 Gy covering less than $20 \%$ of the volume, V $60<40 \%$, $\mathrm{V} 30<80 \%$. For the bladder, the goal was V70 Gy<20\%. The femoral heads were limited to receive 50 Gy in less than $10 \%$ of the volume. The small bowel and sigmoid doses were set $<50 \mathrm{~Gy}$ and $<60 \mathrm{~Gy}$, respectively.

\section{ssIMRT}

Step-and-shoot IMRT plans were generated according to our clinical practice using eight non-coplanar or coplanar beams $\left(225^{\circ}, 260^{\circ}, 295^{\circ}, 330^{\circ}, 30^{\circ}, 65^{\circ}, 100^{\circ}\right.$, $\left.135^{\circ}\right)$. Multiple segments $(80-120)$ were created using the direct machine parameter optimization (DMPO) algorithm of Pinnacle3.

\section{VMAT}

The VMAT plans were generated using two $360^{\circ}$ arcs with the same isocenter rotating clockwise and counter-clockwise starting from $182^{\circ}$ and $178^{\circ}$ with a $10^{\circ}$ collimator angle. Collimator angle was fixed to $10^{\circ}$ to minimize the effects of interleaf leakage and tongueand groove effect. For both the ssIMRT and VMAT plans, the same dose objectives and weightings were used.

The comparison between plans based on analysis of dose volume histograms was performed for V5, V10, V20, V30, V40 of SBM, IBM, and FBM, V20, V30, V40, V70 for bladder, and V30, V40, V76, V80 for the rectum, homogeneity index (homogeneity $\mathrm{MDPD}=\mathrm{MD} /$ PD; MD: Max Dose; PD: prescribed dose), and the monitor units (MU). The two different techniques were statistically compared using a two-tailed pair-wise Wilcoxon signed-ranked test. A value of $\mathrm{p}<0.05$ was considered statistically significant.

\section{Results}

Table 1 displays a comparison of average DVH parameters to SBM, IBM, and FBM receiving 5\%, 10\%, 20\%, $30 \%$, and $40 \%$ receiving of the prescription dose. The average doses, which were achieved by ssIMRT and VMAT, were comparable. V20 values for SBM and FBM were $92.1 \%$ and $74.5 \%$ for ssIMRT, $92.4 \%$ and $74.5 \%$ for VMAT. The V10 and V20 values for IBM is higher with VMAT without statistical significance ( $77.8 \%$ vs. $80.6 \%, \mathrm{p}=0.05$ and $62.3 \%$ vs. $67.2 \%, \mathrm{p}=0.23$, respectively).

The average organs at risk DVHs of bladder and rectum were similar except V80 for rectum, where VMAT was found to be statistically superior to IMRT for V80 that was reduced by $43 \%$ ( $1.6 \%$ vs. $0.9 \%, p=0.01)$.
Table 1 Comparison of average DVH parameters for ssIMRT and VMAT

\begin{tabular}{|c|c|c|c|}
\hline Target volume & ssYART & VMAT & p value \\
\hline \multicolumn{4}{|l|}{ FBM } \\
\hline V5 & \%92.7 & $\% 94.6$ & \\
\hline V10 & $\% 85.2$ & $\% 89.1$ & \\
\hline V20 & $\% 74.5$ & $\% 74.5$ & \\
\hline V30 & $\% 59.5$ & $\% 57.5$ & \\
\hline V40 & $\% 45.7$ & $\% 39.5$ & 0.075 \\
\hline \multicolumn{4}{|l|}{ IBM } \\
\hline V5 & $\% 87.9$ & $\% 88.5$ & \\
\hline V10 & \%77.8 & $\% 80.6$ & \\
\hline V20 & $\% 62.3$ & $\% 67.2$ & \\
\hline V30 & $\% 39.1$ & $\% 41.5$ & \\
\hline V40 & \%18.5 & $\% 16.5$ & 0.141 \\
\hline \multicolumn{4}{|l|}{ SBM } \\
\hline V5 & $\% 100$ & $\% 100$ & \\
\hline V10 & $\% 98.5$ & $\% 98.9$ & \\
\hline V20 & $\% 92.1$ & $\% 92.4$ & \\
\hline V30 & $\% 66.0$ & $\% 68.5$ & \\
\hline V40 & $\% 40.1$ & $\% 37.4$ & 0.109 \\
\hline \multicolumn{4}{|l|}{ Bladder } \\
\hline V20 & \%98.7 & $\% 99.4$ & \\
\hline V30 & $\% 87.0$ & $\% 83.5$ & \\
\hline V40 & $\% 59.7$ & $\% 58.4$ & \\
\hline V70 & \%12.4 & $\% 12.7$ & \\
\hline \multicolumn{4}{|l|}{ Rectum } \\
\hline V30 & \%76.7 & $\% 80.5$ & \\
\hline V40 & $\% 58.3$ & $\% 60.1$ & \\
\hline V76 & \%1.6 & $\% 6.3$ & 0.204 \\
\hline V80 & \%1.6 & $\% 0.9$ & 0.01 \\
\hline Homogenity index & 1.07 & 1.05 & 0.498 \\
\hline Monitor unit & 1591 & 1048 & 0.018 \\
\hline
\end{tabular}

DVH: Dose volume histogram; ssIMRT: Step-and-shoot intensity modulated radiotherapy; VMAT: Volumetric modulated arc therapy; FBM: Femoral bone marrow; IBM: Iliac bone marrow; SBM: Sacral bone marrow

Figure 3 delineates dose distributions of a representative patient for axial, coronal, and sagittal views. The homogeneity index was 1.07 and 1.05 for IMRT and VMAT planning, respectively (Table 1). VMAT provided lower MUs (1591 vs. 1048, $\mathrm{p}=0.01$ ) in comparison to ssIMRT.

\section{Discussion}

We have evaluated the difference of BM sparing in our cohort of patients with high-risk prostate cancer between pelvic VMAT and IMRT plans, and we revealed that VMAT plans without a specific constraint for BM are not found to be superior to ssIMRT in terms of BM reserve irradiation. 

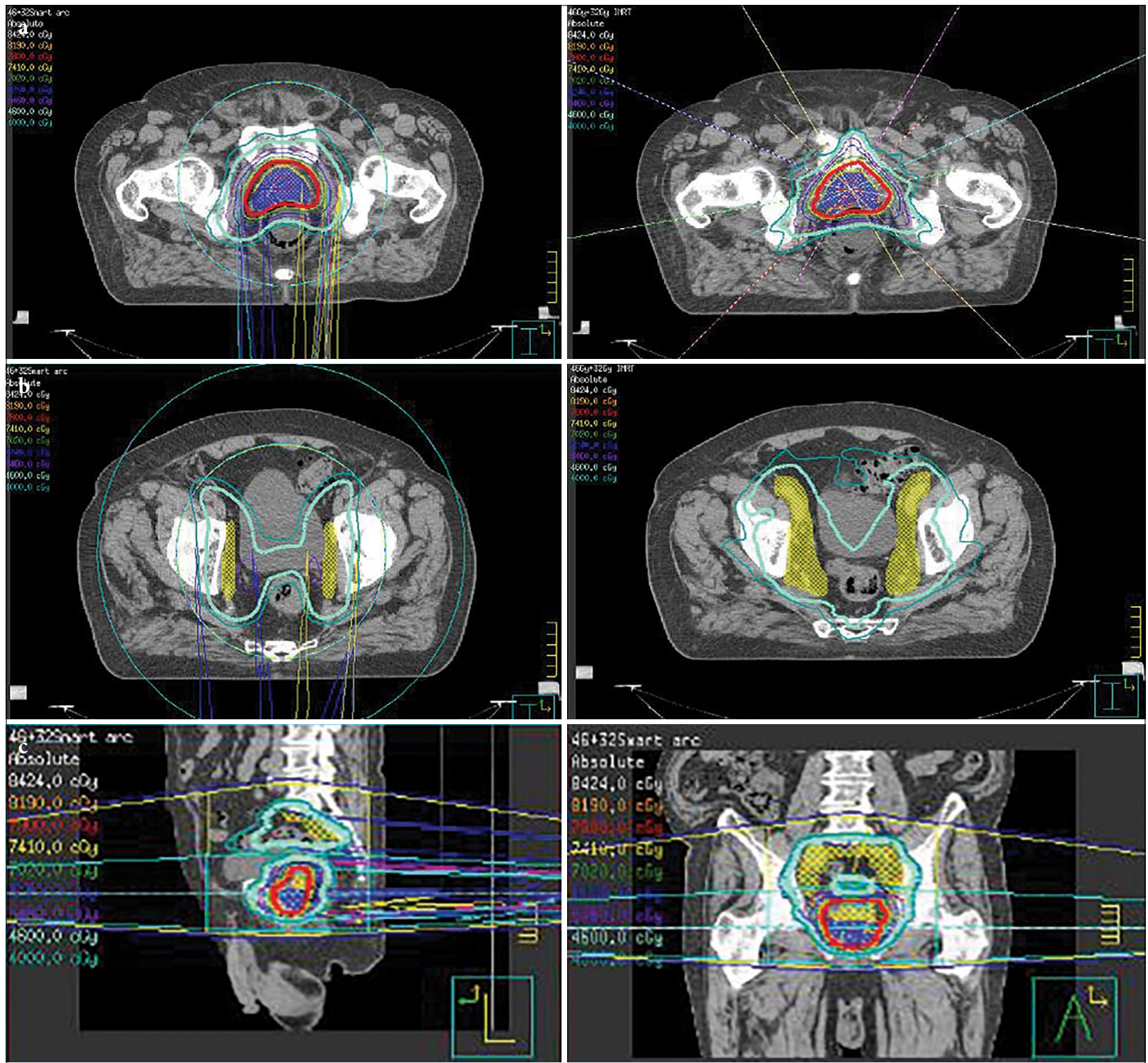

Fig. 3. (a, b) Two different level axial views of pelvis for a representative patient showing isodose lines for VMAT (1) and IMRT (2) plans, (c) coronal and sagittal views of pelvis for the same patient showing isodose lines for VMAT (1) and IMRT (2) plans.

Comparison of VMAT and IMRT in prostate cancer treatment has been ongoing since the last decade. In many studies, five to nine beams IMRT and one or two arc VMAT were in charge.[16-22] VMAT was mostly found to provide similar, slightly better normal organ sparing or superior when compared to IMRT. [16-22] The time period of planning process, the quality parameters specific to planning control steps, and the effort while generating the plan could be significant divergence parameters in these studies. In our cohort, we found VMAT very similar to IMRT for pelvic plus prostate and seminal vesicles irradiation based on the organ at risk parameter limitations except decrement of very high dose volume percentages on rectum (80 Gy). These results are also consistent with the recent meta-analysis reported by Ren et al.[23] In this metaanalysis, V40, V60, and V70 of rectum was significantly decreased by VMAT. On the other hand, no statistical differences were found in V50 of rectum and V40, V50, V60, V70 of bladder. Moreover, VMAT has the edge over IMRT in regards to MUs and treatment time as our study. 
The clinical significance of BM sparing for patients with prostate cancer is yet not known. However, when considering elderly population in prostate cancer, sparing any of the organs at risk would possibly increase the quality of life in long run. Pelvic IMRT or VMAT has been often used for most pelvic malignancies, and despite the ongoing debates, patients with high-risk prostate cancer have been treated with pelvic IMRT or VMAT with one of the highest external radiation doses delivered.[12-14] These VMAT and IMRT novel techniques have been compared in many pelvic tumors as well as for prostate cancer for the ability to reduce the acute and long-term side effects. A great majority of previous dosimetric and clinical studies for prostate cancer focused on the gastrointestinal and genitourinary side effects related with effect on quality of life, but not on $\mathrm{BM}$ reserve. Considering the prolonging overall survival rates, patients with high-risk prostate cancer would have an increased possibly to need chemotherapy at relapse that requires normal hematological parameters; while almost $40 \%$ of the whole body BM reserve located in the pelvic bone is partly in the radiation field during pelvic radiation. In this cohort, we compare routine ssIMRT technique with VMAT to see if there was any improvement in homogeneity, better sparing of BM and other organs at risk. This study was on patients with high-risk prostate cancer, but these results could easily be extrapolated for anal canal cancer, rectum, gynecological cancers, and lymphomas that require pelvic radiotherapy.

Histopathological changes of BM related with radiation can be detected with as low doses as $4 \mathrm{~Gy}$; doses above 10 Gy can lead to progressive changes such as dilated sinusoids, acute hemorrhage, and reduction of precursor cell; and regeneration is lost with 50 Gy or more.[24] Initial efforts evaluating the BM focused on iliac crest sparing that shifted the dose to other pelvic bones shading the BM sparing effect; and Mell et al demonstrated that lowering the V10 and V20 of pelvic $\mathrm{BM}$ was associated with lower grade 2 or more hematological toxicity. $[7,25]$ Lujan et al noted in their cohort that the BM of lumbosacral spine and lower pelvis with femur were strongly associated with hematological toxicity.[24] All previous studies only worked on the prescribed pelvic dose of $45 \mathrm{~Gy}$; and in that range, V20 of BM exceeding the $80 \%$ of the prescribed dose was pointed out to increase the risk of grade 2 or more hematological toxicity by a factor of 4.5. [8] Though we prescribed higher doses with a second phase of cone down to prostate and seminal vesicles up to $78 \mathrm{~Gy}$, both the iliac and lumbosacral doses in our cohort were found to be comparable to the study by Mell et al.
Another aspect could be the dose response relation for secondary cancer, [26] and the risk of leukemia being dose dependent in long-term survivors.[27] Estimated relative risk of leukemia for radiotherapy doses $\geq 15 \mathrm{~Gy}$ is shown to be 7.8 (95\% CI, 1.1-79). [27] Although there is no direct clinical modeling, reduction in BM doses could also translate into risk reduction of leukemia for any pelvic malignancies after pelvic radiotherapy.

Not all parts of the pelvic bone participate in active hematopoiesis, and the percentages in hematopoiesis change based on age. One of the two components of bone is "red" marrow for active hematopoiesis and other is "yellow" BM including predominantly fat. [28] To visualize red marrow, newer techniques such as magnetic resonance spectroscopy or functional single positron emission CT, 18F-Flurodeoxyglucosepositron emission tomography/CT simulation, and MRI by use of quantitative IDEAL IQ were defined in the literature; however, we chose to delineate pelvic bony structures as a simple and classic way to estimate the BM that could be a limitation to define the exact loss in marrow. $[9,29]$ Instead, we tried to use dose cutoff points defined previously to extrapolate the loss.

\section{Conclusion}

In summary, routine VMAT planning without specific dose constraint to BM seems not offering additional benefit to spare BM in comparison to ssIMRT for pelvic plus prostate and seminal vesicles irradiation. This finding could easily be extrapolated to other pelvic malignancies requiring ssIMRT or VMAT in planning, while VMAT sounds safer on rectum based on reduction in high-dose volumes in comparison to IMRT even though both dose volume parameters remain in acceptable clinical limits.

Peer-review: Externally peer-reviewed.

Conflict of Interest: No conflict of interest.

Financial Support: No financial support.

Authorship contributions: Concept - Y.B.; Design - D.S.; Supervision - U.S.; Materials - V.A.; Data collection \&/or processing - D.S.; Analysis and/or interpretation - Y.B.; Literature search - Y.S.; Writing - V.A.; Critical review - U.S.

\section{References}

1. Roach M 3rd, DeSilvio M, Lawton C, Uhl V, Machtay M, Seider MJ, et al. Phase III trial comparing whole- 
pelvic versus prostate-only radiotherapy and neoadjuvant versus adjuvant combined androgen suppression: RadiationTherapy Oncology Group 9413. J Clin Oncol 2003;21(10):1904-11.

2. Lawton CA, DeSilvio M, Roach M 3rd, Uhl V, Kirsch $\mathrm{R}$, Seider M, et al. An update of the phase III trial comparing whole pelvic to prostate onlyradiotherapy and neoadjuvant to adjuvant total androgen suppression: updatedanalysis of RTOG 94-13, with emphasis on unexpected hormone/radiationinteractions. Int J Radiat Oncol Biol Phys 2007;69(3):646-55.

3. Millar J, Boyd R, Sutherland J. An update of the phase III trial comparing whole pelvic to prostate only radiotherapy and neoadjuvant to adjuvant total androgen suppression: updated analysis of RTOG 94-13, with emphasis on unexpected hormone/radiation interactions: in regard to Lawton et al. (Int J Radiat Oncol Biol Phys 2007;69:646-655.). Int J Radiat Oncol Biol Phys 2008;71(1):316; author reply 316.

4. Pommier P, Chabaud S, Lagrange JL, Richaud P, Lesaunier F, Le Prise E, et al. Is there a role for pelvic irradiation in localized prostate adenocarcinoma? Preliminary results of GETUG-01. J Clin Oncol 2007;25(34):5366-73.

5. Mauch P, Constine L, Greenberger J, Knospe W, Sullivan J, Liesveld JL, et al. Hematopoietic stem cell compartment: acute and late effects of radiation therapy and chemotherapy. Int J Radiat Oncol Biol Phys 1995;31(5):1319-39.

6. Mundt AJ, Lujan AE, Rotmensch J, Waggoner SE, Yamada SD, Fleming G, et al. Intensity-modulated whole pelvic radiotherapy in women with gynecologic malignancies. Int J Radiat Oncol Biol Phys 2002;52(5):1330-7.

7. Mell LK, Tiryaki H, Ahn KH, Mundt AJ, Roeske JC, Aydogan B. Dosimetric comparison of bone marrowsparing intensity-modulated radiotherapy versus conventional techniques for treatment of cervical cancer. Int J Radiat Oncol Biol Phys 2008;71(5):1504-10.

8. Albuquerque K, Giangreco D, Morrison C, Siddiqui $\mathrm{M}$, Sinacore J, Potkul R, et al. Radiation-related predictors of hematologic toxicity after concurrent chemoradiation for cervical cancer and implications for bone marrow-sparing pelvic IMRT. Int J Radiat Oncol Biol Phys 2011;79(4):1043-7.

9. Liang Y, Bydder M, Yashar CM, Rose BS, Cornell $\mathrm{M}$, Hoh CK, et al. Prospective study of functional bone marrow-sparing intensity modulated radiation therapy with concurrent chemotherapy for pelvic malignancies. Int J Radiat Oncol Biol Phys 2013;85(2):406-14.

10. Sini C, Fiorino C, Perna L, Noris Chiorda B, Deantoni $\mathrm{CL}$, Bianchi M, et al. Dose-volume effects for pelvic bone marrow in predicting hematological toxicity in prostate cancer radiotherapy with pelvic node irradiation. Radiother Oncol 2016;118(1):79-84.

11. Morikawa LK, Roach M 3rd. Pelvic nodal radiotherapy in patients with unfavorable intermediate and highrisk prostate cancer: evidence, rationale, and future directions. Int J Radiat Oncol Biol Phys 2011;80(1):6-16.

12. Myrehaug S, Chan G, Craig T, Weinberg V, Cheng C, Roach $\mathrm{M}$ 3rd, et al. A treatment planning and acute toxicity comparison of two pelvic nodal volume delineation techniques and delivery comparison of intensity-modulated radiotherapy versus volumetric modulated arc therapy for hypofractionated high-risk prostate cancer radiotherapy. Int J Radiat Oncol Biol Phys 2012;82(4):e657-62.

13. Kopp RW, Duff M, Catalfamo F, Shah D, Rajecki M, Ahmad K. VMAT vs. 7-field-IMRT: assessing the dosimetric parameters of prostate cancer treatment with a 292-patient sample. Med Dosim 2011;36(4):365-72.

14. Davidson MT, Blake SJ, Batchelar DL, Cheung P, Mah $\mathrm{K}$. Assessing the role of volumetric modulated arc therapy (VMAT) relative to IMRT and helical tomotherapy in the management of localized, locally advanced, and post-operative prostate cancer. Int J Radiat Oncol Biol Phys 2011;80(5):1550-8.

15. Lawton CA, Michalski J, El-Naqa I, Buyyounouski MK, Lee WR, Menard C, et al. RTOG GU Radiation oncology specialists reach consensus on pelvic lymph node volumes for high-risk prostate cancer. Int J Radiat Oncol Biol Phys 2009;74(2):383-7.

16. Quan EM, Li X, Li Y, Wang X, Kudchadker RJ, Johnson JL, et al. A comprehensive comparison of IMRT and VMAT plan quality for prostate cancer treatment. Int J Radiat Oncol Biol Phys 2012;83(4):1169-78.

17. Wolff D, Stieler F, Welzel G, Lorenz F, Abo-Madyan Y, Mai S, et al. Volumetric modulated arc therapy (VMAT) vs. serial tomotherapy, step-and-shoot IMRT and 3D-conformal RT for treatment of prostate cancer. Radiother Oncol 2009;93(2):226-33.

18. Bedford JL. Treatment planning for volumetric modulated arc therapy. Med Phys 2009;36(11):5128-38.

19. Boylan CJ, Golby C, Rowbottom CG. A VMAT planning solution for prostate patients using a commercial treatment planning system. Phys Med Biol 2010;55(14):N395-404.

20. Kinhikar RA, Pawar AB, Mahantshetty U, Murthy V, Dheshpande DD, Shrivastava SK. Rapid Arc, helical tomotherapy, sliding window intensity modulated radiotherapy and three dimensional conformal radiation for localized prostate cancer: a dosimetric comparison. J Cancer Res Ther 2014;10(3):575-82.

21. Yoo S, Wu QJ, Lee WR, Yin FF. Radiotherapy treatment plans with RapidArc for prostate cancer involving seminal vesicles and lymph nodes. Int J Radiat Oncol Biol Phys 2010;76(3):935-42. 
22. Khan MI, Jiang R, Kiciak A, Ur Rehman J, Afzal M, Chow JC. Dosimetric and radiobiological characterizations of prostate intensity-modulated radiotherapy and volumetric-modulated arc therapy: A single-institution review of ninety cases. J Med Phys 2016;41(3):162-8.

23. Ren W, Sun C, Lu N, Xu Y, Han F, Liu YP, et al. Dosimetric comparison of intensity-modulated radiotherapy and volumetric-modulated arc radiotherapy in patients with prostate cancer: a meta-analysis. J Appl Clin Med Phys 2016;17(6):254-62.

24. Lujan AE, Mundt AJ, Yamada SD, Rotmensch J, Roeske JC. Intensity-modulated radiotherapy as a means of reducing dose to bone marrow in gynecologic patients receiving whole pelvic radiotherapy. Int J Radiat Oncol Biol Phys 2003;57(2):516-21.

25. Mell LK, Schomas DA, Salama JK, Devisetty K, Aydogan B, Miller RC, et al. Association between bone marrow dosimetric parameters and acute hematologic toxicity in anal cancer patients treated with concurrent chemotherapy and intensity-modulated radiotherapy. Int J Radiat Oncol Biol Phys 2008;70(5):1431-7.

26. Schneider U, Sumila M, Robotka J, Gruber G, Mack A, Besserer J. Dose-response relationship for breast cancer induction at radiotherapy dose. Radiat Oncol 2011;6:67.

27. Travis LB, Andersson M, Gospodarowicz M, van Leeuwen FE, Bergfeldt K, Lynch CF, Treatment-associated leukemia following testicular cancer. J Natl Cancer Inst 2000;92(14):1165-71.

28. Vande Berg BC, Malghem J, Lecouvet FE, Maldague B. Magnetic resonance imaging of the normal bone marrow. Skeletal Radiol 1998;27(9):471-83.

29. Ai J, Xie T, Sun W, Liu Q. Red bone marrow dose calculations in radiotherapy of prostate cancer based on the updated VCH adult male phantom. Phys Med Biol 2014;59(7):1815-30. 